\title{
A prediction model of postdonation renal function using dynamic kidney computed tomography volumetry in living kidney donor
}

\author{
Seoungjun Lim ${ }^{1}$, Jieun Kwon², Youngmin Ko', Hyeeun Kwon, Joohee Jung ${ }^{1}$, Hyunwook Kwon ${ }^{1}$, Younghoon Kim¹, \\ Kyowon Lee ${ }^{2}$, Sung Shin ${ }^{1}$
}

\footnotetext{
${ }^{1}$ Department of Surgery-Transplantation, Asan Medical Center, University of Ulsan College of Medicine, Seoul, Korea

${ }^{2}$ Department of Surgery-Transplantation, Samsung Medical Center, Seoul, Korea
}

Background: The risk of renal failure after live kidney donation can be predicted based on various clinical information. We tried introduce a model to predict the risk of renal failure after live kidney donation using predonation variables including residual kidney volume proportion (RKP, remnant kidney volume/total kidney volume) measured by computed tomography (CT) volumetry. Methods: Patients who had donor nephrectomy between May 2007 and December 2019 in two independent centers. Age, sex, body mass index (BMI), hypertension, smoking history, preoperative estimated glomerular filtration rate (eGFR) and RKP were investigated. Primary end point is eGFR less than $60 \mathrm{~mL} / \mathrm{min} / \mathrm{m}^{2} 6$ months after kidney donation. Univariable and multivariable analysis was performed using the Cox hazard regression model.

Results: A total of 1,628 and 690 live kidney donors were included in training and validation cohort, respectively. The eGFR was less than $60 \mathrm{~mL} / \mathrm{min} / \mathrm{m}^{2} 6$ months after kidney donation in 235 donors (14.4\%) of the training cohort and 178 donors $(25.8 \%)$ of the validation cohort. In univariable analysis, sex, age, BMI, hypertension, preoperative eGFR, and remnant kidney proportion were significantly associated with the primary end point. After multivariable analysis, the variables used in the score system of the prediction model were sex, age, eGFR, and remnant kidney proportion. The sum of score ranges from 0 to 10 (sex: male, 1 ; female, 0), (age at operation: $<30,0 ; 30-39,1 ; 40-59,2 ; \geq 60$, 3), (preoperative eGFR: $\geq 100,0 ; 90-99,2 ; 80-89,4 ;<80,5)$, (RKP: $\geq 52$, $0 ;<52,1)$. This prediction model for the primary end point showed good discrimination (training: AUC, $0.891 ; 95 \%$ confidence interval [CI], 0.868-0.913; validation: AUC, $0.865 ; 95 \% \mathrm{Cl}, 0.833-0.897$ ) and calibration by Hosmer-Lemeshow goodness-of-fit test (training: $\chi^{2}=1.9912, \mathrm{df}=8, \mathrm{P}=0.9813$; validation: $\chi^{2}=47.335, \mathrm{df}=8, \mathrm{P}=1.323 \mathrm{e}-07$ ).

Conclusions: Our prediction model based on dynamic CT volumetry and clinical data may be useful to estimate the risk of significant renal dysfunction after kidney donation.

Corresponding author: Sung Shin

E-mail:sshin@amc.seoul.kr

(c) The Korean Society for Transplantation

This is an Open Access article distributed under the terms of the Creative Commons Attribution Non-Commercial License (http://creativecommons.org/licenses/by-nc/4.0/) which permits unrestricted non-commercial use, distribution, and reproduction in any medium, provided the original work is properly cited. 